\title{
Helmholtz algebraic solitons
}

\section{J M Christian ${ }^{1,3}$, G S McDonald ${ }^{1}$, and P Chamorro-Posada ${ }^{2}$}

1 Joule Physics Laboratory, School of Computing, Science and Engineering, Materials \& Physics Research Centre, University of Salford, Salford M5 4WT, UK

2 Departamento de Teoría de la Señal y Comunicaciones e Ingeniería Telemática, Universidad de Valladolid, ETSI Telecomunicación, Campus Miguel Delibes s/n, 47011 Valladolid, Spain

\begin{abstract}
We report, to the best of our knowledge, the first exact analytical algebraic solitons of a generalized cubic-quintic Helmholtz equation. This class of governing equation plays a key role in photonics modelling, allowing a full description of the propagation and interaction of broad scalar beams. New conservation laws are presented, and the recovery of paraxial results is discussed in detail. The stability properties of the new solitons are investigated by combining semi-analytical methods and computer simulations. In particular, new general stability regimes are reported for algebraic bright solitons.
\end{abstract}

PACS number(s): 42.65.-k (nonlinear optics), 42.65.Tg (optical solitons), 42.65.Wi (nonlinear waveguides), 05.45.Yv (solitons)

Submitted to Journal of Physics A: Mathematical and Theoretical as a Paper.

$9^{\text {th }}$ December 2009

\footnotetext{
${ }^{3}$ Corresponding author.
} 


\section{Introduction}

Solitons are robust, self-localizing waves that can exist in a system when linear spreading effects are opposed by nonlinearity [1]. Their prevalence in mathematical physics is largely due to a relatively small set of universal equations governing a wide range of systems [2]. Solitons tend to be constructed from hyperbolic functions (such as sech and tanh) of the space and/or time coordinates, and are thus exponentially localized wavepackets. However, universal equations can often also support algebraic solitons - particular solutions that are constructed from rational functions. Such solutions are less tightly localized than their hyperbolic counterparts [3]; their tails fall off with a power-law distribution, i.e., algebraically.

Perhaps the simplest universal equations with algebraic soliton solutions are of the modified Korteweg-de Vries (KdV) type [4]. KdV-type models, for example, underpin Fermi-Pasta-Ulam descriptions of lattice dynamics [5]. Algebraic solitons are encountered in fluid mechanics as solutions to the Davey-Stewartson [6] and Benjamin-Ono [7] equations. Deep water waves and ion-acoustic waves in plasmas can be described by algebraic solitons of the derivative-nonlinear Schrödinger (NLS) [8] and the Kadomtsev-Petviashvili equations [2,3,9]. In photonics, algebraic solitons occur in such contexts as Raman scattering [10], self-induced transparency [11] (Maxwell-Bloch-type systems), pulse propagation in dispersive fibres [12] (derivative-NLS), electromagnetic modes of planar waveguides [13] (dual power-law NLS), and solitary-wave polaritons [14] (Boussinesq equation). Coupled modes and periodic systems can also support KdV- and NLS-type algebraic "gap solitons," respectively [15]. Finally, the Klein-Gordon models in $\phi^{4}-\phi^{6}$ theories of particle physics also admit algebraic solutions [16]. This brief summary aims to illustrate that algebraic solitons are fundamental excitations in nonlinear science.

In this paper, we are especially interested in the seminal works by Hayata and Koshiba (who derived the first dual power-law NLS algebraic solitons) [13], and Micallef et al (who later showed that these solitons arise mathematically from a particular limit of a hyperbolic solution family) [17]. We report what we believe to be the first algebraic solitons for a nonlinear Helmholtz (NLH) equation. NLH-type models are also universal, appearing whenever the Laplacian is present, e.g., in fluidic, plasma, acoustic, and optical nonlinear contexts. Here we consider spatial solitons in uniform twodimensional planar waveguides, though our general results also have a wider mathematical appeal. A spatial soliton is a stationary beam that can emerge as a dominant electromagnetic mode when diffractive broadening (linear spreading) is exactly balanced by self-lensing (a nonlinear change in the local refractive index of the host medium) [18].

\section{Helmholtz soliton theory}

\subsection{The role of Helmholtz equations}

Helmholtz equations play a fundamental role in photonics modelling. They provide a platform for describing any experimental arrangement that exploits broad beams in off-axis contexts. It turns out that even the most fundamental "building block" optical geometries have intrinsically angular characters. A pertinent example is the multiplexing of two or more beams at arbitrary angles (with respect to the reference direction) and orientations (with respect to each other). Another example is material interface effects, where beam incidence, transmission and reflection angles at the boundary between dissimilar media may be of arbitrary magnitude.

While paraxial theory well describes the small-angle limit of scalar multiplexing [19] and interface [20] configurations, only recently have their arbitrary-angle properties been explored in detail [21,22]. These recent analyses relied upon detailed knowledge of the exact analytical soliton solutions to the governing Helmholtz equations. Such models are suitable for addressing the issue of obliquepropagation effects because they respect a fundamental symmetry: in uniform media, there is no distinction between the spatial dimensions. For example, in two-dimensional planar waveguides, the transverse and longitudinal directions are physically equivalent. This spatial symmetry is absent from paraxial theory, and Helmholtz angular corrections to key predictions may exceed $100 \%$. 


\subsection{Field and envelope equations}

In Helmholtz modelling [23,24], one tends to adopt the scalar approximation whereby the beam waist $w_{0}$ is assumed to be much larger than the free-space carrier wavelength $\lambda$. Order-of-magnitude corrections to the governing equation, which arise from the polarization-scrambling term $\nabla(\nabla \cdot \mathbf{E})$ in Maxwell's equations [25-27], are thus unnecessary. Such corrections are routinely based upon a single parameter-of-smallness, $\varepsilon \equiv \lambda / w_{0}$, and are necessary when $\varepsilon \sim O(1)$. Here, we consider only those contexts where the inequality $\varepsilon<<O(1)$ is always rigorously satisfied.

For a continuous-wave scalar electric field $\tilde{E}(x, z, t)=E(x, z) \exp (-\mathrm{i} \omega t)+$ c.c. with angular frequency $\omega$, and where $E(x, z)$ satisfies the Maxwell field equation [23,24], one has

$$
\left(\frac{\partial^{2}}{\partial z^{2}}+\frac{\partial^{2}}{\partial x^{2}}\right) E(x, z)+\frac{\omega^{2} n^{2}}{c^{2}} E(x, z)=0 .
$$

The spatial coordinates, $x$ and $z$, appear with equal status so that diffraction is fully two-dimensional (i.e., occurring in the transverse and longitudinal directions). The refractive index is taken to be $n(|E|)=$ $n_{0}+n_{N L}(|E|)$, where $n_{0}$ is the linear index at frequency $\omega, n_{N L}(|E|)=-n_{\sigma}|E|^{\sigma}+n_{2 \sigma}|E|^{2 \sigma}$ is the fielddependent part, $n_{\sigma}$ and $n_{2 \sigma}$ are small positive constants, and the exponent $\sigma>0$. This classic type of dual power-law distribution appears frequently in photonics; for instance, one might interpret it as an approximation of a quite general model for saturation, namely $n_{N L}(|E|)=-n_{\sigma}|E|^{\sigma} /\left[1+\left(n_{2 \sigma} / n_{\sigma}\right)|E|^{\sigma}\right]$. Various choices of the parameter set $\left(n_{\sigma}, n_{2 \sigma}, \sigma\right)$ capture Kerr [18,19], single power-law [28], cubicquintic [29], and quadratic-cubic [14] nonlinearities. Many authors have studied this model in its most general form [13,17,30-32]. With advances in materials science and fabrication, it may one day be possible to tailor dielectric media with arbitrary values of $\sigma$ for a whole range of information communication and technology applications.

For a weak optical nonlinearity, where $\left|n_{N L}(|E|)\right|<<n_{0}$, one has that $n^{2}(|E|) \simeq n_{0}^{2}+2 n_{0} n_{N L}(|E|)$. To facilitate comparison with paraxial theory, $z$ is chosen to be the longitudinal (reference) direction, and $E(x, z)$ is expressed as $E(x, z)=E_{0} u(x, z) \exp (\mathrm{i} k z)$. Without further approximation, one can derive the envelope equation,

$$
\kappa \frac{\partial^{2} u}{\partial \zeta^{2}}+\mathrm{i} \frac{\partial u}{\partial \zeta}+\frac{1}{2} \frac{\partial^{2} u}{\partial \xi^{2}}-\alpha|u|^{\sigma} u+\gamma|u|^{2 \sigma} u=0 .
$$

Here, $\zeta=z / L_{D}$ and $\xi=2^{1 / 2} x / w_{0}$, where $L_{D}=k w_{0}{ }^{2} / 2$ is the diffraction length of a reference Gaussian beam, $k=n_{0} k_{0}$ is the wavenumber of the carrier wave, and $k_{0}=\omega / c=2 \pi / \lambda$. The inverse beam-width is quantified by $\kappa \equiv 1 /\left(k w_{0}\right)^{2}=\varepsilon^{2} / 4 \pi^{2} n_{0}^{2}<<O(1)$. Finally, the parameters $\alpha$ and $\gamma$ are related to the constant $E_{0}$. A convenient normalization that could be adopted is $E_{0} \equiv\left(n_{0} / n_{\sigma} L_{D} k\right)^{1 / \sigma}$, so that $\alpha=1$ and $\gamma=$ $E_{0}{ }^{\sigma}\left(n_{2 \sigma} / n_{\sigma}\right)$. For mathematical completeness, however, both $\alpha$ and $\gamma$ will be retained in the presented solutions. The corresponding paraxial model $[13,17]$ can be recovered by neglecting the first term in equation (2), which is just the slowly-varying envelope approximation (SVEA).

\section{Helmholtz bright solitons}

The full generality of the $\partial_{z z}$ operator has been preserved in equations (1) and (2). For instance, both models are bi-directional and thus support forward- and backward-propagating fields. It is important to note that forward and backward beams are distinguishable only by their propagation direction with respect to the reference axis. In all other respects, the solutions are physically identical to each other since they are related through a $180^{\circ}$ rotation. We now show that equation (2) possesses a variety of exact analytical solutions.

\subsection{Hyperbolic solitons}

Equation (2) admits two families of exact analytical hyperbolic bright soliton: 


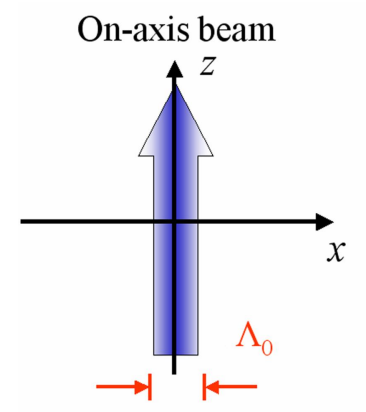

(a) $\theta=0^{\circ}$

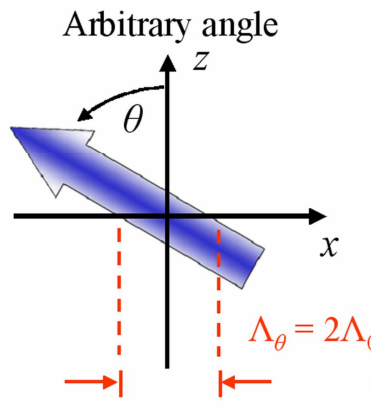

(b) $\theta=+60^{\circ}$

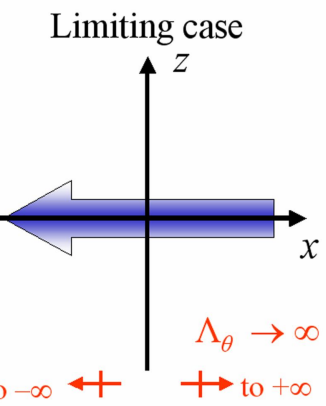

(c) $\theta=+90^{\circ}$

Figure 1. (Colour online) Schematic diagram illustrating the geometry of a forwardpropagating Helmholtz soliton. (a) The on-axis beam whose width in the $(x, z)$ frame is $\Lambda_{\theta}=$ $\Lambda_{0}$. (b) During oblique propagation at angle $\theta$, the projected beam width is given by $\Lambda_{\theta}=$ $\Lambda_{0} \sec \theta=2 \Lambda_{0}$ when $|\theta|=60^{\circ}$ (to scale). (c) In the extreme case of $|\theta|=90^{\circ}$, the beam appears to be infinitely broad when observed from the $(x, z)$ frame.

$$
\begin{aligned}
& u(\xi, \zeta)=\eta_{h}\left[A_{h} \cosh \left(\sigma \sqrt{2 \beta} \frac{\xi+V \zeta}{\sqrt{1+2 \kappa V^{2}}}\right)-1\right]^{-1 / \sigma} \\
& \times \exp \left[ \pm \mathrm{i} \sqrt{\frac{1+4 \kappa \beta}{1+2 \kappa V^{2}}}\left(-V \xi+\frac{\zeta}{2 \kappa}\right)\right] \exp \left(-\mathrm{i} \frac{\zeta}{2 \kappa}\right) \text {, } \\
& A_{h} \equiv\left[1+\frac{(2+\sigma)^{2}}{1+\sigma}\left(\frac{\gamma}{\alpha^{2}}\right) \beta\right]^{1 / 2} \\
& \eta_{h} \equiv\left(\frac{2+\sigma}{\alpha} \beta\right)^{1 / \sigma} .
\end{aligned}
$$

The beam width measured by an observer in the $(x, z)$ frame is $\Lambda=\left(1+2 \kappa V^{2}\right)^{1 / 2} \Lambda_{0}$, where $\Lambda_{0} \equiv$ $(1 / \sigma)(2 \beta)^{-1 / 2}$ and $V$ is the conventional transverse velocity parameter. The forward solution (upper sign) describes an exponentially-localized beam propagating at an angle $\theta=\tan ^{-1}\left[(2 \kappa)^{1 / 2} V\right]$ with respect to the $+z$ direction, where $-\infty \leq V \leq+\infty$ corresponds to $-90^{\circ} \leq \theta \leq+90^{\circ}$ (this beam is shown schematically in figure 1); the backward solution (lower sign) describes a similar beam evolving in the opposite direction. Solution (3) is characterized by the internal parameter $\beta$, whose physical significance will shortly become clear.

\subsection{Algebraic solitons}

Two families of algebraic soliton can be obtained by taking the limit $\beta \rightarrow 0$ in the hyperbolic solutions (3):

$$
\begin{aligned}
& u(\xi, \zeta)=\eta_{a}\left[a^{2}\left(\frac{\xi+V \zeta}{\sqrt{1+2 \kappa V^{2}}}\right)^{2}+1\right]^{-1 / \sigma} \exp \left[ \pm \mathrm{i} \frac{1}{\sqrt{1+2 \kappa V^{2}}}\left(-V \xi+\frac{\zeta}{2 \kappa}\right)\right] \exp \left(-\mathrm{i} \frac{\zeta}{2 \kappa}\right), \\
& \eta_{a} \equiv\left[\left(\frac{2 \alpha}{\gamma}\right)\left(\frac{1+\sigma}{2+\sigma}\right)\right]^{1 / \sigma},
\end{aligned}
$$




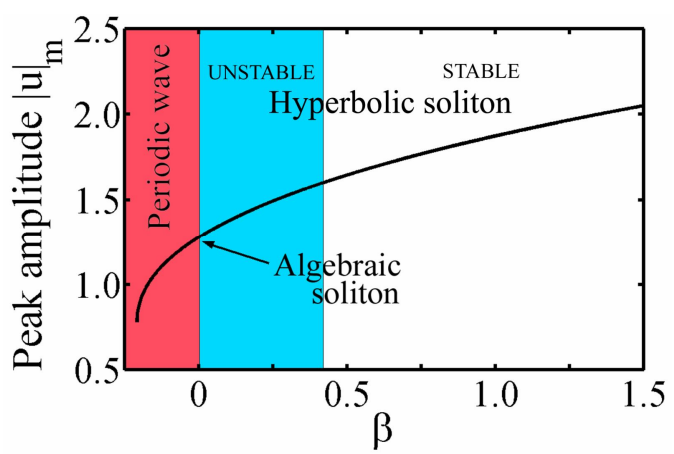

Figure 2. (Colour online) Peak amplitude $|u|_{\mathrm{m}}$ of solution (3) as a function of $\beta$ for $\sigma=1.4$ (within the conditionally stable regime, as discussed in section 4). $\beta>0$ corresponds to hyperbolic soliton (3), and $\beta=0$ to algebraic soliton (4). The hyperbolic soliton is unstable against small perturbations when $0<\beta<\beta_{\text {min }} \simeq 0.42$ (blue shaded area). The domain $\beta<0$ (red shaded area) is accessed through analytic continuation, where one finds a transverselyperiodic wave. Other parameters: $\alpha=\gamma=1$.

$$
a^{2} \equiv\left(\frac{2 \alpha^{2}}{\gamma}\right) \frac{\sigma^{2}(1+\sigma)}{(2+\sigma)^{2}} \text {. }
$$

Solution (4) is determined uniquely for any choice of material parameters $(\alpha, \gamma, \sigma)$; its amplitude profile is classified as "Lorentzian" when $\sigma=1$, "sub-Lorentzian" when $\sigma<1$, and "super-Lorentzian" when $\sigma$ $>1$. The algebraic bright soliton (4) is weakly localized, with relatively slow power-law asymptotics, $|u(\xi, \zeta)| \sim|\xi+V \zeta|^{-2 / \sigma}$ as $|\xi+V \zeta| \rightarrow \infty$. The beam becomes more localized as $\sigma$ decreases, and such narrowing is off-set by an increase in the peak amplitude. This relationship follows directly from the nature of a solitary wave: any increase in diffraction must be balanced by an increase in self-focusing.

Theoretical modelling is ultimately concerned with physical phenomena in the laboratory [i.e., the $(x, z)]$ frame. To this end, it is desirable to be able to move easily from scaled to unscaled quantities and coordinates. Such transformations between Helmholtz equations (1) and (2) are fully self-consistent i.e., exact in their handling of the phase and propagation angle of the beam - since the generality of the (in-plane) Laplacian, $\nabla^{2} \equiv \partial_{z z}+\partial_{x x}$, is maintained. In contrast, such transformations can be hindered by the SVEA, where the longitudinal phase shift is always implicitly approximated.

In the $(x, z)$ frame, the longitudinal phase shift $\Delta \phi$ accrued by the hyperbolic soliton (3) during propagation from $z=z_{1}$ to $z=z_{2}$ is

$$
\Delta \phi=k_{0} n_{0} \cos \theta(1+4 \kappa \beta)^{1 / 2} \Delta z
$$

where $\Delta z \equiv z_{2}-z_{1}$. When $\beta \rightarrow 0$, one has that $\Delta \phi \sim k_{0} n_{0} \cos \theta \Delta z$, and the phase shift is then identical to that picked up by a plane wave propagating in a purely-linear medium with refractive index $n_{0}$. It is in this sense that algebraic solitons have been interpreted as the threshold for linear wave propagation (i.e., where the carrier wave of the soliton doesn't 'see' the nonlinearity) [17]. The relationship between algebraic soliton (4) and the linear-wave threshold clearly involves the phase in the laboratory reference frame, so an exact transition from hyperbolic to algebraic solutions, valid across the entire range of propagation angles, requires a Helmholtz description.

Analytic continuation of $\beta$ into the domain $\beta<0$ can yield delocalized waves, whose amplitude profiles are periodic in the transverse direction $[\operatorname{since} \cosh (i \Theta)=\cos \Theta]$. The forward and backward periodic waves are given by 

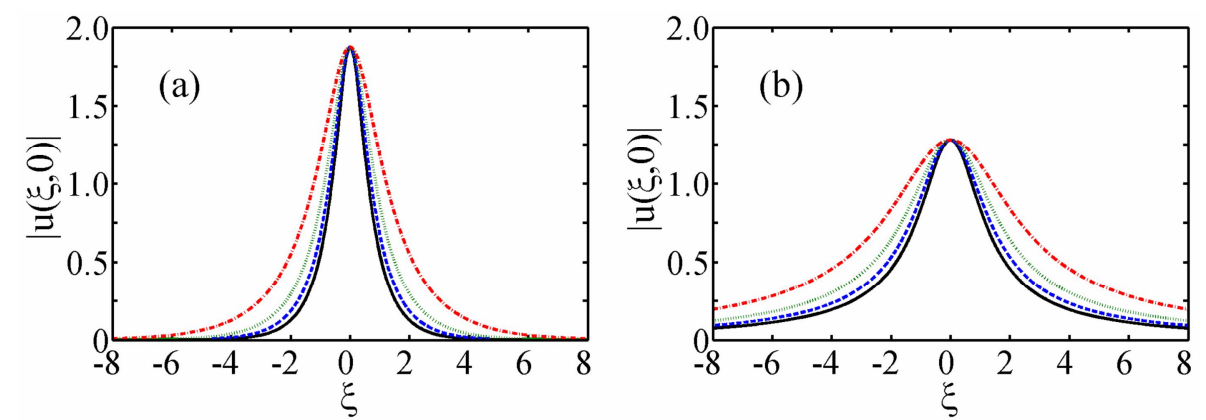

Figure 3. (Colour online) Angular beam broadening effect. (a) Hyperbolic soliton (3) with $\beta=$ 1. (b) Algebraic bright soliton (4), obtained in the limit that $\beta \rightarrow 0$. Solid line (black): $\theta=0^{\circ}$ (the paraxial profile); dashed line (blue): $|\theta|=30^{\circ}$; dotted line (green): $|\theta|=45^{\circ}$; dot-dash line (red): $|\theta|=60^{\circ}$ (where the beam width appears to have doubled, relative to its on-axis value). Other parameters: $\sigma=1.4$ and $\alpha=\gamma=1$.

$$
\begin{aligned}
u(\xi, \zeta)=\eta_{p}\left[A_{p} \cos (\right. & \left.\left.\sigma \sqrt{2|\beta|} \frac{\xi+V \zeta}{\sqrt{1+2 \kappa V^{2}}}\right)-1\right]^{-1 / \sigma} \\
& \times \exp \left[ \pm \mathrm{i} \sqrt{\frac{1-4 \kappa|\beta|}{1+2 \kappa V^{2}}}\left(-V \xi+\frac{\zeta}{2 \kappa}\right)\right] \exp \left(-\mathrm{i} \frac{\zeta}{2 \kappa}\right),
\end{aligned}
$$

where $A_{p} \equiv\left(1-|\beta| /|\beta|_{\max }\right)^{1 / 2},|\beta|_{\max } \equiv(1+\sigma)\left(\alpha^{2} / \gamma\right) /(2+\sigma)^{2}$ and $\eta_{p} \equiv[-(2+\sigma)|\beta| / \alpha]^{1 / \sigma}$. This solution exists provided $0<|\beta|<|\beta|_{\max }$. Propagation (as opposed to evanescence) of the periodic wave requires $|\beta|<1 / 4 \kappa$. This condition places a physical limit on the smallest transverse period of nonlinear wavetrains in relation to the optical wavelength; such considerations are absent from paraxial theory [17]. In parameter regimes of interest, for example, $\alpha=1, \gamma \leq O(1), \sigma=O(1)$ and $\kappa<<O(1)$, one finds that the first of these two conditions is nearly always satisfied before the second comes into play (we also note that the second inequality has no analogue in paraxial theory). The connection between hyperbolic, algebraic, and periodic waves is illustrated in figure 2.

\subsection{Spatial symmetry properties}

The symmetry between a forward beam and its backward counterpart can be made explicit by combining the two beams into a single solution. The trigonometric relations $\cos \theta=1 /\left(1+2 \kappa V^{2}\right)^{1 / 2}$ and $\sin \theta=$ $(2 \kappa)^{1 / 2} V /\left(1+2 \kappa V^{2}\right)^{1 / 2}$ allow one to eliminate $V$ so that the hyperbolic soliton (3) can be written as

$$
\begin{aligned}
u(\xi, \zeta)=\eta_{h}\left\{A_{h} \cosh [\right. & \left.\left.\sigma \sqrt{2 \beta}\left(\xi \cos \theta+\frac{\zeta}{\sqrt{2 \kappa}} \sin \theta\right)\right]-1\right\}^{-1 / \sigma} \\
& \times \exp \left[i \sqrt{\frac{1+4 \kappa \beta}{2 \kappa}}\left(-\xi \sin \theta+\frac{\zeta}{\sqrt{2 \kappa}} \cos \theta\right)\right] \exp \left(-\mathrm{i} \frac{\zeta}{2 \kappa}\right)
\end{aligned}
$$

In a similar way, the algebraic soliton (4) becomes

$$
\begin{aligned}
u(\xi, \zeta)=\eta_{a}\left[a^{2}(\xi \cos \theta\right. & \left.\left.+\frac{\zeta}{\sqrt{2 \kappa}} \sin \theta\right)^{2}+1\right]^{-1 / \sigma} \\
& \times \exp \left[\mathrm{i} \frac{1}{\sqrt{2 \kappa}}\left(-\xi \sin \theta+\frac{\zeta}{\sqrt{2 \kappa}} \cos \theta\right)\right] \exp \left(-\mathrm{i} \frac{\zeta}{2 \kappa}\right) .
\end{aligned}
$$


The propagation angle appearing in solutions $(7 a)$ and $(7 b)$ now satisfies $-180^{\circ} \leq \theta \leq+180^{\circ}$, while the remaining parameters are unchanged. One can also re-express the pair of periodic solutions (6) in this type of symmetric form.

Oblique evolution is a potentially dominant Helmholtz contribution since the beam broadening factor $\left(1+2 \kappa V^{2}\right)^{1 / 2}=\sec \theta$ is unbounded: it may be of any order irrespective of $\kappa$ and the system nonlinearity. For example, the moderate angle $|\theta|=60^{\circ}$ implies that $2 \kappa V^{2}=3$, and an observer in the $(x, z)$ frame thus perceives the beam width to have doubled relative to its on-axis value (see figure 3 ). As $|\theta| \rightarrow$ $90^{\circ}$, one has that $2 \kappa V^{2} \rightarrow \infty$ and the beam appears to be infinitely broad when viewed from the $(x, z)$ frame (where propagation takes place perpendicularly to the reference direction). This geometrical property of Helmholtz solutions appears in the delocalized wave (6) as an increase in the spatial period $\Gamma_{\theta}$, where $\Gamma_{\theta}=\left(1+2 \kappa V^{2}\right)^{1 / 2} \Gamma_{0}$ and $\Gamma_{0} \equiv 2 \pi /\left[\sigma(2 \beta)^{1 / 2}\right]$. Off-axis effects alone can thus define a scenario in which the angular nonparaxial correction can assume any order $0<2 \kappa V^{2} \leq \infty$ (equivalent to $0<|\theta|$ $\leq 90^{\circ}$ ) while the narrowbeam inequality $\kappa \simeq \varepsilon^{2}<<O(1)$ is always fully satisfied.

\subsection{Conservation laws}

Using quite general field-theoretic techniques [33], one can derive three conserved quantities associated with equation (2) that represent the energy-flow, momentum, and Hamiltonian, respectively:

$$
\begin{aligned}
& W=\int_{-\infty}^{+\infty} d \xi\left[|u|^{2}-i \kappa\left(u^{*} \frac{\partial u}{\partial \zeta}-u \frac{\partial u^{*}}{\partial \zeta}\right)\right], \\
& M=\int_{-\infty}^{+\infty} d \xi\left[\frac{\mathrm{i}}{2}\left(u^{*} \frac{\partial u}{\partial \xi}-u \frac{\partial u^{*}}{\partial \xi}\right)-\kappa\left(\frac{\partial u^{*}}{\partial \zeta} \frac{\partial u}{\partial \xi}+\frac{\partial u^{*}}{\partial \xi} \frac{\partial u}{\partial \zeta}\right)\right], \\
& H=\int_{-\infty}^{+\infty} d \xi\left[\frac{1}{2} \frac{\partial u^{*}}{\partial \xi} \frac{\partial u}{\partial \xi}-\kappa \frac{\partial u^{*}}{\partial \zeta} \frac{\partial u}{\partial \zeta}+\alpha \frac{|u|^{2+\sigma}}{1+\frac{1}{2} \sigma}-\gamma \frac{|u|^{2(1+\sigma)}}{1+\sigma}\right],
\end{aligned}
$$

By writing solution (3) as $u(\xi, \zeta)=F(s) \exp [\mathrm{i} \phi(\xi, \zeta)]$, where $F$ is the (real) amplitude distribution and $s \equiv$ $(\xi+V \zeta) /\left(1+2 \kappa V^{2}\right)^{1 / 2}$, the integrals in equations $(8 a)-(8 c)$ can be expressed more compactly:

$$
\begin{aligned}
& W= \pm(1+4 \kappa \beta)^{1 / 2} P \\
& M=\frac{V}{\sqrt{1+2 \kappa V^{2}}}[(1+4 \kappa \beta) P-2 \kappa Q] \\
& H=\frac{W}{2 \kappa}-\frac{1}{\sqrt{1+2 \kappa V^{2}}}\left(\frac{1}{2 \kappa}\right)[(1+4 \kappa \beta) P-2 \kappa Q] .
\end{aligned}
$$

The quantities $P$ and $Q$ are given by

$$
\begin{aligned}
& P \equiv \int_{-\infty}^{+\infty} d s F^{2}(s)=\frac{2}{\sqrt{2 \beta}}\left(\frac{\eta_{h}^{2}}{\sigma}\right) \int_{0}^{+\infty} d y\left[A_{h} \cosh (y)-1\right]^{-2 / \sigma} \\
& Q \equiv \int_{-\infty}^{+\infty} d s\left[\frac{d}{d s} F(s)\right]^{2}=2 \sqrt{2 \beta}\left(\frac{\eta_{h}^{2} A_{h}^{2}}{\sigma}\right) \int_{0}^{+\infty} d y \sinh ^{2}(y)\left[A_{h} \cosh (y)-1\right]^{-(2+2 / \sigma)} .
\end{aligned}
$$


The upper (lower) sign in equation (9a) denotes the energy-flow invariant for the forward (backward) beam. By taking the limit $\beta \rightarrow 0$ and substituting for the algebraic soliton (4), it can be shown that

$$
P=\sqrt{\pi}\left(\frac{\eta_{a}^{2}}{a}\right) \frac{\Gamma(2 / \sigma-1 / 2)}{\Gamma(2 / \sigma)}
$$

and

$$
Q=\sqrt{\pi}\left(\frac{2 \eta_{a}^{2} a}{\sigma(2+\sigma)}\right) \frac{\Gamma(2 / \sigma+1 / 2)}{\Gamma(2 / \sigma+1)}
$$

where $\Gamma$ denotes the Gamma function and $0<\sigma<4$. Interestingly, Helmholtz bright solitons are also found to satisfy the free-particle relationship $\partial H / \partial M=\partial_{V} H / \partial_{V} M=V$, where $\partial_{V} \equiv \partial / \partial V$. Aside from their physical importance, the integrals in equations (8) allow one to monitor the integrity of the algorithm used to solve equation (2) numerically [34].

\subsection{The paraxial limit}

The corresponding paraxial model [13,17] can be obtained from equation (2) by invoking the SVEA, whereby the operator $\kappa \partial_{\zeta \zeta}$ is neglected. It is therefore intuitive that when $\kappa \partial_{\zeta \zeta} \rightarrow 0$, all Helmholtz contributions to beam evolution are negligible, and one should uncover the predictions of paraxial theory. This type of recovery procedure is more subtle than simply setting $\kappa=0$. Instead, one is obliged to consider a simultaneous multiple limit [35].

To recover the paraxial solution of Micallef et al [17] from hyperbolic soliton (3), one must allow $\kappa \rightarrow 0$ (broad beam), $\kappa \beta \rightarrow 0$ (moderate nonlinear phase shift), and $\kappa V^{2} \rightarrow 0$ (negligible propagation angle). We first consider the asymptotic behaviour of the forward solutions, where $\theta \rightarrow 0^{\circ}$. When applied to the hyperbolic soliton, the triple limit leads to

$$
u(\xi, \zeta) \sim \eta_{h}\left\{A_{h} \cosh [\sigma \sqrt{2 \beta}(\xi+V \zeta)]-1\right\}^{-1 / \sigma} \exp \left[-\mathrm{i} V \xi+\mathrm{i}\left(\beta-\frac{V^{2}}{2}\right) \zeta\right]
$$

The $\beta$ parameter can thus be identified with the on-axis longitudinal phase shift in the corresponding paraxial solution. A similar convergence of the Helmholtz algebraic soliton (4) to its paraxial counterpart requires $\kappa \rightarrow 0$ and $\kappa V^{2} \rightarrow 0$, so that

$$
u(\xi, \zeta) \sim \eta_{a}\left[a^{2}(\xi+V \zeta)^{2}+1\right]^{-1 / \sigma} \exp \left(-\mathrm{i} V \xi-\mathrm{i} \frac{V^{2}}{2} \zeta\right)
$$

By applying the multiple limit to the conserved quantities in equations (9), one obtains the familiar paraxial invariants $W \sim P, M \sim V P$ and $H \sim \frac{1}{2} V^{2} P-\beta P+Q$ for the hyperbolic soliton (the algebraic solution requires $\beta \rightarrow 0$ in the expression for $H$ ) [31]. For the backward beams, where $|\theta| \rightarrow 180^{\circ}$, application of the same multiple limit yields

$$
u(\xi, \zeta) \sim \eta_{h}\left\{A_{h} \cosh [\sigma \sqrt{2 \beta}(\xi+V \zeta)]-1\right\}^{-1 / \sigma} \exp \left[\mathrm{i} V \xi-\mathrm{i}\left(\beta-\frac{V^{2}}{2}\right) \zeta\right] \exp \left(-\mathrm{i} 2 \frac{\zeta}{2 \kappa}\right)
$$

and

$$
u(\xi, \zeta) \sim \eta_{a}\left[a^{2}(\xi+V \zeta)^{2}+1\right]^{-1 / \sigma} \exp \left(\mathrm{i} V \xi+\mathrm{i} \frac{V^{2}}{2} \zeta\right) \exp \left(-\mathrm{i} 2 \frac{\zeta}{2 \kappa}\right)
$$



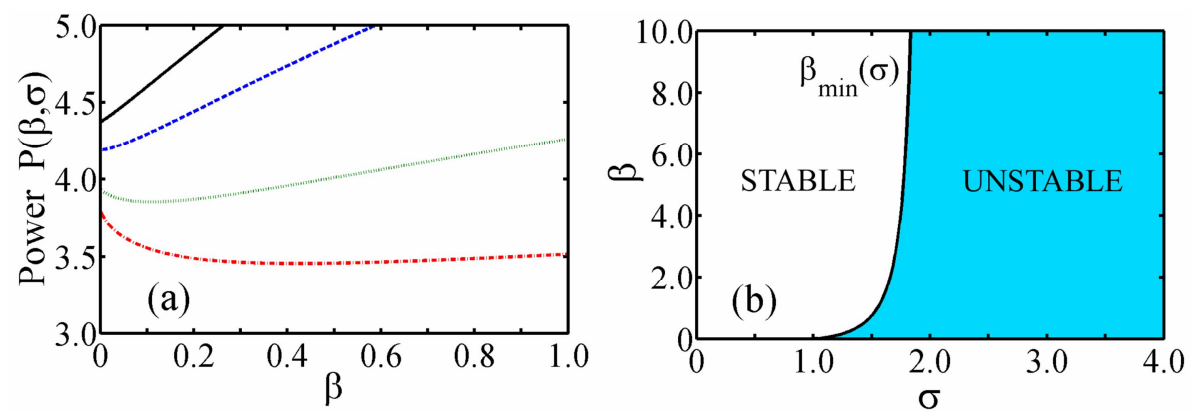

Figure 4. (Colour online) Stability characteristics of hyperbolic solitons. (a) Variation of the beam power $P$ with $\beta$ [solid line (black): $\sigma=0.9$; dashed line (blue): $\sigma=1.0$; dotted line (green): $\sigma=1.2$; dot-dash line (red): $\sigma=1.4$ ]. For $0<\sigma \leq 1$, the solutions are unconditionally stable $(d P / d \beta>0$ for all $\beta \geq 0)$. For $1<\sigma<2$, the solutions are conditionally stable, so that $d P / d \beta>0$ only when $\beta>\beta_{\min }(\sigma)$. (b) The boundary between stable and unstable solutions in the $(\sigma, \beta)$ plane is determined (numerically) by the curve $\beta_{\min }(\sigma)$.

respectively, while the invariants become $W \sim-P, M \sim V P$, and $H \sim \frac{1}{2} V^{2} P-3 \beta P+Q-P / \kappa$. Since the latter set of results retain $\kappa$-dependent contributions, it is clear that backward fields have no analogue in paraxial theory. This confirms the fact that paraxial models can support wave propagation in a single longitudinal direction only.

\section{Stability of Helmholtz bright solitons}

Linear analysis has predicted that plane-wave solutions to NLH equations with arbitrary dispersive nonlinearity functions are modulationally stable in the same parameter regions as their paraxial counterparts [36]. However, the stability of localized solutions against arbitrary perturbations is a much more interesting problem: such stability is a key property of solitons. Without loss of generality, we set $\alpha=\gamma=1$ throughout this section.

\subsection{Analysis}

The stability of paraxial bright solitons (11a) has been studied by Micallef et al [17] using the wellknown Vakhitov-Kolokolov (VK) criterion [30,37]. Spatial symmetry allows the same criterion to predict the stability properties of Helmholtz solitons [36,38]. This is because an isolated off-axis beam can always be observed from the "on-axis" frame of reference by means of a rotation of the coordinate axes. In the on-axis frame, where $V=\kappa V^{2}=0$, beams with $\kappa<<O(1)$ and $\kappa \beta \ll O(1)$ are quasi-paraxial since the forward solution (3) exhibits only an $O(\kappa)$ correction to the longitudinal phase shift.

The VK criterion states that bright solitons can be stable against small perturbations if $d P / d \beta>0$, where

$$
P(\beta ; \sigma) \equiv \int_{-\infty}^{+\infty} d \xi|u(\xi, \zeta ; \beta ; \sigma)|^{2}
$$

[we note, in passing, that equations (12) and (9d) are formally identical for paraxial solitons]. Satisfaction of the VK criterion is a necessary but not sufficient condition for stability [36]; simulations are essential to establish the robustness of solutions against arbitrarily-large perturbations. Hyperbolic solitons are predicted to be unconditionally stable when $0<\sigma \leq 1$ since, for that range of $\sigma$, the VK inequality $d P / d \beta>0$ is satisfied for any $\beta \geq 0$. However, figure $4(a)$ reveals that when $1<\sigma<2$, the slope $d P / d \beta>0$ only when $\beta$ exceeds a minimum value, denoted by $\beta_{\min }$. Regions of stability in the ( $\sigma$, $\beta$ ) plane are thus separated by a boundary represented by the curve $\beta_{\min }(\sigma)$ [see figure $4(b)$ ]. When $\sigma \geq$ 2 , the VK criterion predicts that the soliton is always unstable since $d P / d \beta<0$ for $\beta \geq 0$. 

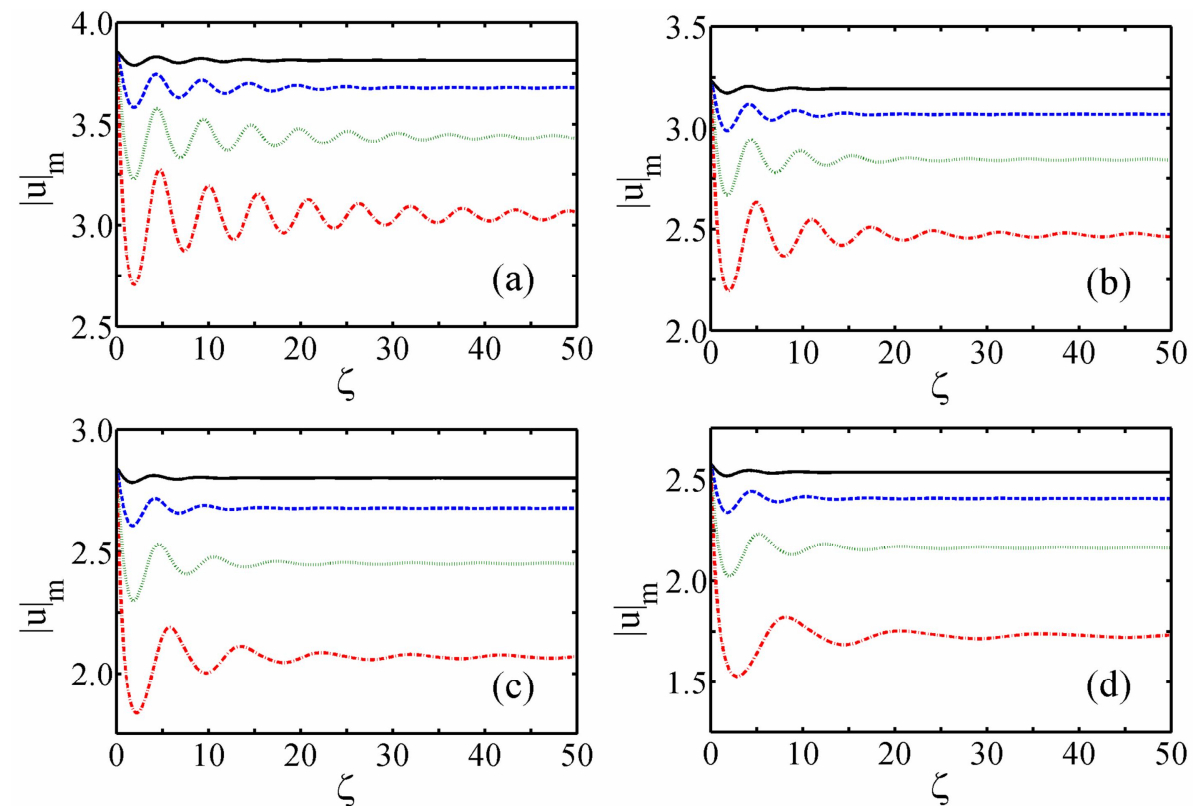

Figure 5. (Colour online) Evolution of the peak amplitude $|u|_{m}$ of a perturbed hyperbolic soliton with $\beta=1$ when $(a) \sigma=0.5,(b) \sigma=0.6$, (c) $\sigma=0.7$ and $(d) \sigma=0.8$. Solid lines (black): $|\theta|$ $=10^{\circ}$; dashed lines (blue): $|\theta|=20^{\circ}$; dotted lines (green): $|\theta|=30^{\circ}$; dot-dash lines (red): $|\theta|=$ $40^{\circ}$.

\subsection{Hyperbolic solitons}

The stability of hyperbolic solitons is considered through the perturbed input beam

$$
u(\xi, 0)=\eta_{h}\left[A_{h} \cosh (\sigma \sqrt{2 \beta} \xi)-1\right]^{-1 / \sigma} \exp \left(-\mathrm{i} V \sqrt{\frac{1+4 \kappa \beta}{1+2 \kappa V^{2}}} \xi\right)
$$

whose launching angle is $\theta=\tan ^{-1}\left[(2 \kappa)^{1 / 2} V\right]$. By applying a rotational transformation [39], one can see that the initial condition (13) is equivalent to an on-axis Helmholtz soliton whose width has been reduced by a factor of $\left(1+2 \kappa V^{2}\right)^{1 / 2}=\sec \theta$. For $\kappa=10^{-3}\left(\kappa=10^{-4}\right)$, launching angles of $|\theta|=10^{\circ}, 20^{\circ}$, $30^{\circ}$ and $40^{\circ}$ correspond to transverse velocities of $|V| \simeq 3.94,8.14,12.91$ and $18.76(|V| \simeq 12.47$, $25.74,40.82$ and 59.33), respectively. These angles are clearly non-trivial and lie outside the scope of paraxial theory.

In the unconditionally stable domain $(0<\sigma \leq 1)$, evolution is generally characterized by monotonically-decreasing oscillations in the beam parameters (amplitude, width, and area $=$ amplitude $\times$ width). These oscillations are accompanied by a small amount of radiation, and they disappear as $\zeta \rightarrow \infty$ to leave a stationary state (see figure 5). Solitons with $0<\sigma \leq 1$ can thus generally be interpreted as stable fixed-point attractors: the emission of radiation throughout reshaping provides a mechanism for local dissipation while the system remains globally conservative [36,38]. As discussed in the preceding subsection, there should be no instability in the range $0<\sigma \leq 1$ (as prescribed by the VK inequality). However, simulations have revealed that as $\sigma \rightarrow 1$, sufficiently large perturbations can induce a diffractive instability whereby the amplitude of the beam tends to zero as $\zeta \rightarrow \infty$.

To gain insight into the propagation properties of conditionally-stable hyperbolic solitons (where 1 $<\sigma<2)$, it is instructive to recognize that the power $P_{\text {in }}(\beta, \sigma, V)$ of the perturbed input beam (13) is related to the power $P(\beta, \sigma)$ of the unperturbed beam by

$$
P_{\text {in }}(\beta, \sigma ; V)=\frac{P(\beta, \sigma)}{\sqrt{1+2 \kappa V^{2}}} \equiv P(\beta, \sigma) \cos \theta,
$$



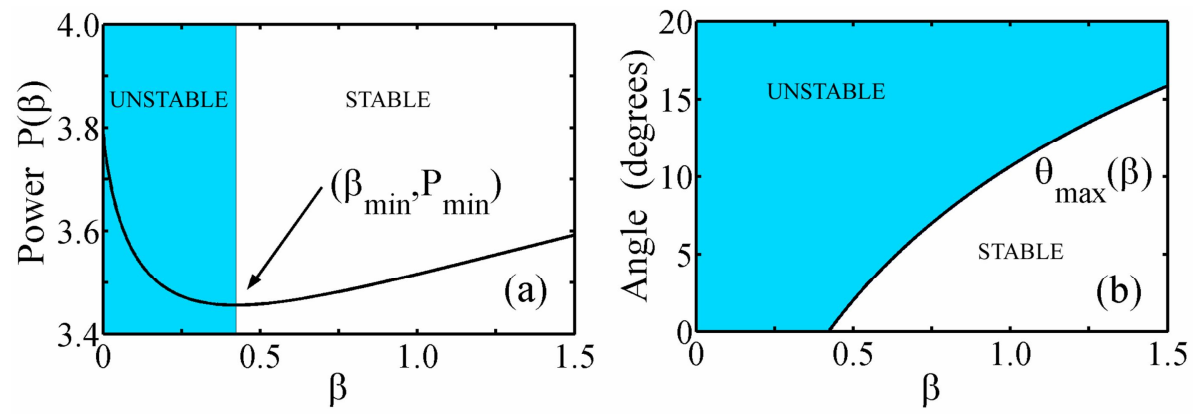

Figure 6. (Colour online) (a) Beam power calculated from equation (12) for hyperbolic soliton (3) with $\sigma=1.4$, where $\left(\beta_{\min }, P_{\min }\right) \simeq(0.42,3.46)$. The criterion $d P / d \beta>0$ is met when $\beta>$ $\beta_{\min }$ (unshaded region). (b) Theoretical prediction from equation (15) for the maximum launching angle of input beam (13) before the onset of instability, where $P_{\text {in }} \leq P_{\min }$.

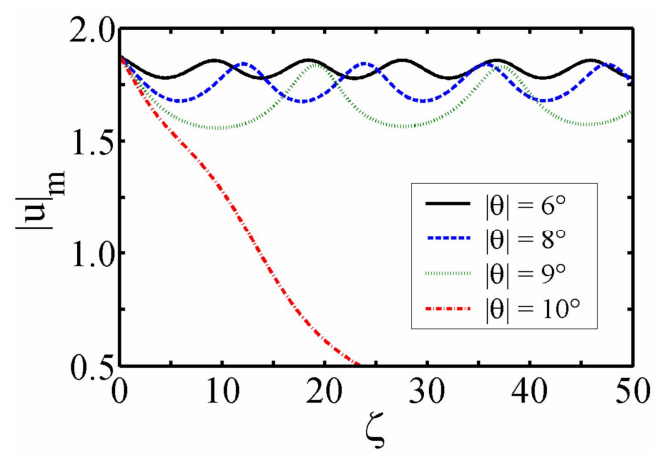

Figure 7. (Colour online) Long-lived self-persistent reshaping oscillations in the peak amplitude $|u|_{\mathrm{m}}$ of a perturbed (conditionally-stable) hyperbolic soliton (3) with $\sigma=1.4$. For $\beta=1$, equation (15) predicts that $|\theta|_{\max } \simeq 10.7^{\circ}$. Instability sets in when $|\theta|$ is slightly less than the theoretical value of $|\theta|_{\max }$.

where $P$ is given by equation (12). That is, $|\theta|>0$ decreases the power of the input beam relative to its unperturbed value (i.e., relative to the power of the exact solution with the same parameters). Figure 4(a) suggests there is a minimum power $P_{\min }$ that can sustain a propagating soliton; when $P_{\text {in }}<P_{\min }$, one expects that no stationary states exist and that the input beam will transform into radiation modes [30]. Thus, $\left(\beta_{\min }, P_{\min }\right)$ are the coordinates of the local minimum in the $P(\beta)$ curve [see figure $6(a)$ ]. One can then expect to encounter a maximum perturbation, against which the soliton is stable, through the following condition: $P_{\mathrm{in}}=P_{\min }$ when $|\theta|=|\theta|_{\max }$. It can then be shown that for any input beam with $\beta>$ $\beta_{\min }$,

$$
\tan \left(|\theta|_{\max }\right)=\left[\left(\frac{P}{P_{\min }}\right)^{2}-1\right]^{1 / 2} .
$$

Equation (15) assumes that the energy of the input and asymptotic beams are identical, and that radiation shed during reshaping can be neglected. Regions of predicted stability are illustrated in figure $6(b)$ for $\sigma=1.4$. The simulations shown in figure 7 are in good agreement with equation (15), though instability sets in when $|\theta|$ is slightly less than the theoretical $|\theta|_{\max }$. This difference indicates that, as might be expected, stronger radiation shedding can come into play when the perturbed soliton approaches the instability threshold (the fraction of energy transferred to radiation modes will depend upon system parameters). Below the instability threshold (i.e., $|\theta|<|\theta|_{\max }$ so that $P_{\text {in }}>P_{\min }$ ), perturbed solitons undergo long-lived self-sustained oscillations in their parameters; we classify such solitons as stable limit-cycle attractors [36,38]. These quasi-periodic orbits are effectively internal modes, and they have been analysed by Pelinovsky et al [30]. As the threshold is approached (i.e., $|\theta| \rightarrow|\theta|_{\max }$, or $P_{\text {in }} \rightarrow P_{\min }$ ) one 

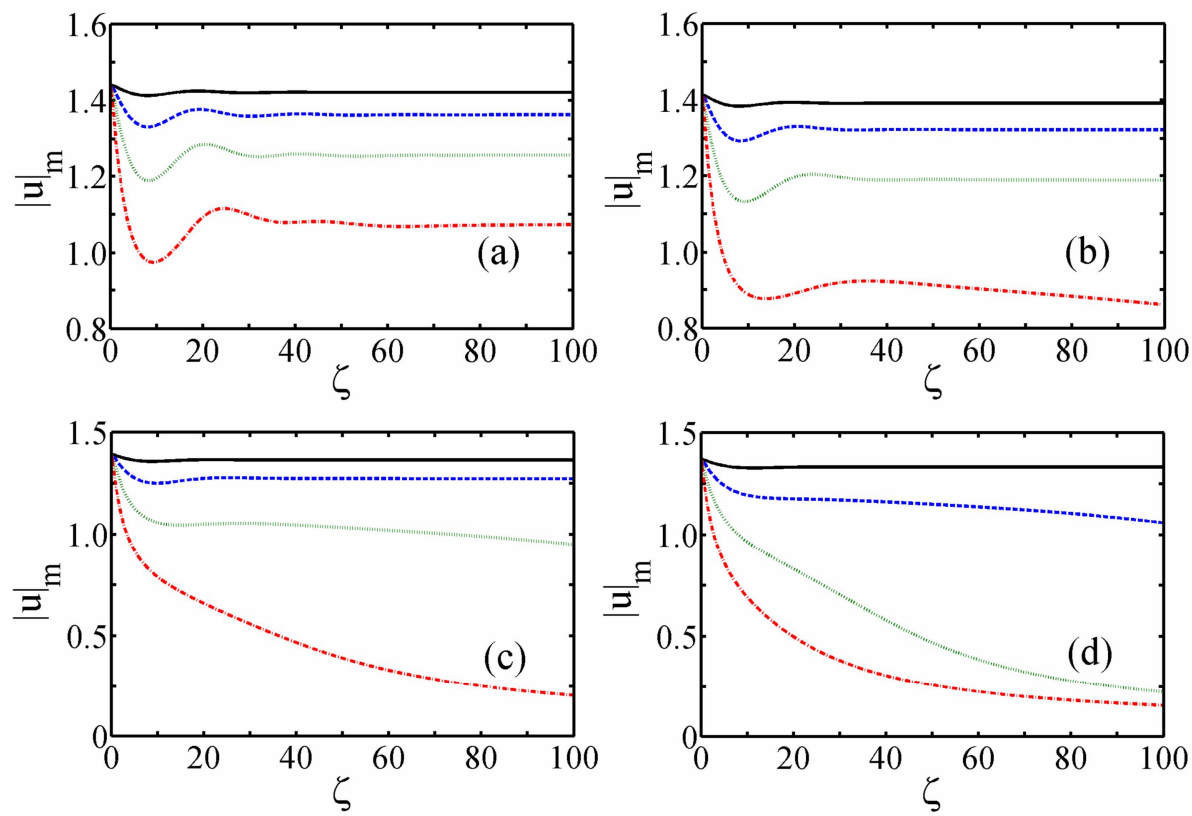

Figure 8. (Colour online) Evolution of the peak amplitude $|u|_{\mathrm{m}}$ of a perturbed algebraic bright soliton when $(a) \sigma=0.5$, (b) $\sigma=0.6,(c) \sigma=0.7$ and $(d) \sigma=0.8$. Solid lines: $|\theta|=10^{\circ}$; dashed lines: $|\theta|=20^{\circ}$; dotted lines: $|\theta|=30^{\circ}$; dot-dash lines: $|\theta|=40^{\circ}$.

finds that the evolving soliton diffracts toward a zero-amplitude state (see figure 7).

\subsection{Algebraic solitons}

Analysing the stability of algebraic solitons is a notoriously difficult task. Conventional nonlinearperturbative techniques tend to become frustrated in all but the simplest cases because of their relatively slow asymptotics (i.e., power-law instead of exponential) [30]. Some insight can be gained from inspection of figure 4(a). For example, in the region where the hyperbolic solutions are unconditionally stable $(0<\sigma \leq 1)$, the beam power has a positive slope (i.e., $d P / d \beta>0)$ at $\beta=0$. However, when $\sigma>$ 1 , one finds that $d P / d \beta<0$ at $\beta=0$. Thus, algebraic solitons are expected to be always unstable when $\sigma>1$. Micallef et al [17] suggested that paraxial algebraic solitons $(11 b)$ are inherently unstable due to the absence of an arbitrary internal parameter. Their simulations confirmed that, even when $0<\sigma \leq 1$, algebraic solitons are weakly unstable. Pelinovsky et al attributed this instability to resonant interactions with infinitely long linear waves [30].

We now undertake a fully nonlinear (i.e., numerical) stability analysis of Helmholtz algebraic solitons through consideration of the input beam

$$
u(\xi, 0)=\eta_{a}\left(a^{2} \xi^{2}+1\right)^{-1 / \sigma} \exp \left(-\mathrm{i} \frac{V}{\sqrt{1+2 \kappa V^{2}}} \xi\right) .
$$

Initial condition (16) corresponds to launching solution (4) without the beam-broadening factor $(1+$ $\left.2 \kappa V^{2}\right)^{1 / 2}$. Analysis predicts that when $P_{\text {in }}<P$ (i.e., $|\theta|>0$ ), any perturbed algebraic soliton with $0<\sigma<$ 4 will transform into radiation modes [30]. However, our simulations have shown that when $\sigma$ is sufficiently less than 1, this collapse may be suppressed. The reshaping properties of algebraic solitons can thus be reminiscent of those of their hyperbolic counterparts. Figure 8 shows that as $\sigma \rightarrow 1$, the stability of algebraic solitons gradually diminishes. For instance, the solution in media characterized by $\sigma=$ 0.5 is robust against all four increasingly-strong angular perturbations [see figure $8(a)$ ]; the solution with $\sigma=0.8$ is robust only against the weakest perturbation [see figure $8(d)$ ]. 

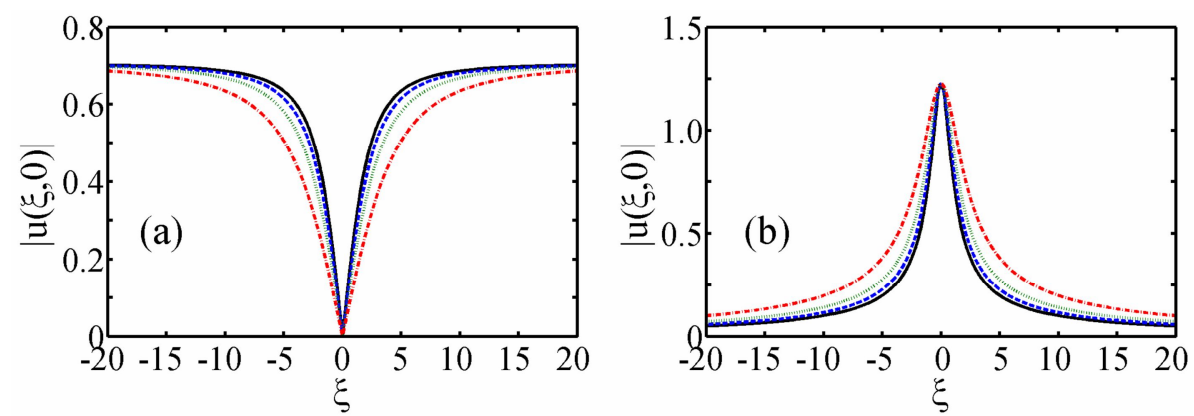

Figure 9. (Colour online) Comparison of the angular beam broadening effect for the algebraic dark soliton (17) [(a)] with that of the corresponding (i.e., $\sigma=2)$ bright soliton (4) [(b)]. As $\xi$ $\rightarrow \pm \infty$, one finds that the dark solution behaves as $u \sim \pm v / a$ (reflecting the $\pi$ phase shift). Solid line (black): $\theta=0^{\circ}$ (the paraxial profile); dashed line (blue): $|\theta|=30^{\circ}$; dotted line (green): $|\theta|=45^{\circ}$; dot-dashed line (red): $|\theta|=60^{\circ}$. Other parameters: $\alpha=\gamma=1$.

\section{Helmholtz algebraic dark solitons}

\subsection{Exact analytical solutions}

Equation (2) permits the existence of algebraic dark solitons in the particular case of a cubic-quintic nonlinearity (i.e., when $\sigma=2$ ). In symmetric form,

$$
\begin{aligned}
u(\xi, \zeta)=v(\xi \cos \theta & \left.+\frac{\zeta}{\sqrt{2 \kappa}} \sin \theta\right)\left[a^{2}\left(\xi \cos \theta+\frac{\zeta}{\sqrt{2 \kappa}} \sin \theta\right)^{2}+1\right]^{-1 / 2} \\
\times & \exp \left[\mathrm{i} \sqrt{\frac{1+4 \kappa \mu}{2 \kappa}}\left(-\xi \sin \theta+\frac{\zeta}{\sqrt{2 \kappa}} \cos \theta\right)\right] \exp \left(-\mathrm{i} \frac{\zeta}{2 \kappa}\right),
\end{aligned}
$$

where $a^{2} \equiv \alpha^{2} / 6 \gamma, v \equiv(3 / \alpha)^{1 / 2} a^{2}$ and $\mu \equiv-\alpha^{2} / 4 \gamma$. Like its bright counterpart [solution (4)], the dark solution is specified uniquely by the choice of $\alpha$ and $\gamma$. There is a phase shift of $\pi$ radians across the transverse extent of the field, and an absolute-zero in the field at the beam centre (see figure 9). However, the solution is structurally distinct from the more familiar phase-topological dark solitons [40,41]. In passing, we note an asymmetry between algebraic solutions (17) and (4) [in the case of $\sigma=2$ ] while the intensity profiles of canonical bright and black Kerr solitons, $I_{\mathrm{b}}=\operatorname{sech}^{2}(s)$ and $I_{\mathrm{d}}=\tanh ^{2}(s)$, are related by $I_{\mathrm{d}}=1-I_{\mathrm{b}}$, the same type of relationship does not hold for bright and dark algebraic beams.

One can now consider the multiple limit $\kappa \rightarrow 0, \kappa \mu \rightarrow 0$ and $\kappa V^{2} \rightarrow 0$. From the forward solution, one can recover the paraxial soliton of Hayata and Koshiba [13], namely

$$
u(\xi, \zeta) \sim v(\xi+V \zeta)\left[a^{2}(\xi+V \zeta)^{2}+1\right]^{-1 / 2} \exp \left[-\mathrm{i} V \xi+\mathrm{i}\left(\mu-\frac{V^{2}}{2}\right) \zeta\right]
$$

The backward solution tends to

$$
u(\xi, \zeta) \sim v(\xi+V \zeta)\left[a^{2}(\xi+V \zeta)^{2}+1\right]^{-1 / 2} \exp \left[\mathrm{i} V \xi-\mathrm{i}\left(\mu-\frac{V^{2}}{2}\right) \zeta\right] \exp \left(-\mathrm{i} 2 \frac{\zeta}{2 \kappa}\right)
$$

which has no counterpart in paraxial theory due to the rapid-phase $\kappa$-dependent term that survives the limit. It is interesting to note that equation (2) supports both bright and dark algebraic solitons without needing to reverse the relative signs of the nonlinear terms. 


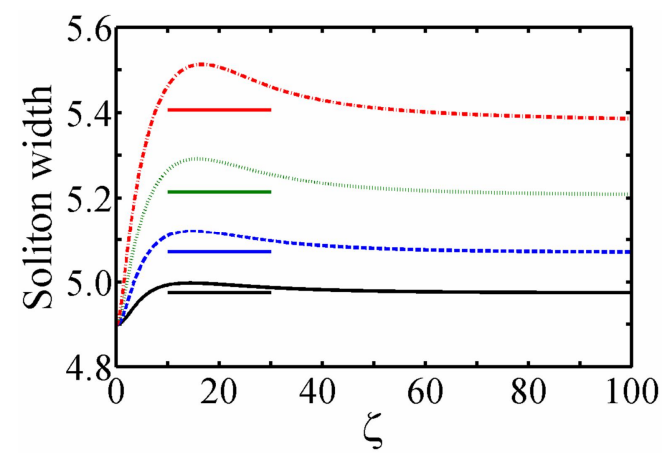

Figure 10. (Colour online) Evolution of the beam full-width of a perturbed algebraic dark soliton. The width tends asymptotically toward the value $\Lambda_{\infty} \sim\left(1+2 \kappa V^{2}\right)^{1 / 2} \Lambda_{0}$, where $\Lambda_{0} \equiv$ $2 / a$ (horizontal bars). Solid line (black): $|\theta|=10^{\circ}$; dashed line (blue): $|\theta|=15^{\circ}$; dotted line (green): $|\theta|=20^{\circ}$; dot-dash line (red): $|\theta|=25^{\circ}$. The widths have been calculated by fitting the numerical data to the nonlinear refractive-index function $n_{N L}=-\alpha|u|^{2}+\gamma|u|^{4}$ in combination with solution (17). For larger values of $|\theta|$, the evolving beam radiates more strongly and suffers fluctuations to its shape that can complicate interpolation. Other parameters: $\alpha=\gamma=$ 1 .

\subsection{Numerical stability analysis}

The apparent absence of a suitable stability criterion has so far rendered an in-depth analysis of solution (17) problematic. For instance, one cannot apply the renormalized-momentum integral [42] since there is no intrinsic-velocity parameter [41]. One also encounters divergences in the integral conserved quantities [equations $(8 a)-(8 c)$ ] because the solution does not break up in the way the renormalization method demands (i.e., a plane-wave background modulated by an obliquely-propagating grey "dip").

Here, we investigate the stability properties of Helmholtz algebraic dark solitons numerically using the input beam

$$
u(\xi, 0)=v \xi\left(a^{2} \xi^{2}+1\right)^{-1 / 2} \exp \left(-\mathrm{i} V \sqrt{\frac{1-4 \kappa \beta}{1+2 \kappa V^{2}}} \xi\right)
$$

This initial condition corresponds to launching an exact paraxial soliton (that does not account for the beam-broadening factor). The full width of the beam is found to tend towards an asymptotic value $\Lambda_{\infty}=(2 / a)\left(1+2 \kappa V^{2}\right)^{1 / 2}$ as $\zeta \rightarrow \infty$, eventually leaving a stationary beam (see figure 10). Simulations show that the dark beam can be robust against perturbations, even through its bright counterpart [solution (4) with $\sigma=2$ ] is always unstable. Thus, one can interpret the dark solitons as fixed-point attractors. Similar qualitative behaviour has been uncovered in the propagation properties of Helmholtz Kerr dark solitons [41].

\section{Conclusions}

We have presented a variety of new exact analytical solutions to a generalized cubic-quintic nonlinear Helmholtz equation, including hyperbolic and algebraic solitons, and transversely-periodic waves. The mathematical origin of the bright algebraic family lies in taking a particular limit of the hyperbolic family. We have shown that a fully self-consistent transition from a hyperbolic soliton into an algebraic soliton can be achieved using the Helmholtz formalism. New conservation laws have been reported. The conserved quantities have been evaluated exactly for algebraic solitons, and a classical particle energy-momentum relationship has been uncovered for Helmholtz solitons. Well-known paraxial results $[13,17]$ have also emerged from a quite general limit process. The stability of Helmholtz bright solitons has been investigated by combining conventional semi-analytical techniques [30,37] with beam geometry in the $(x, z)$ frame [35,39], and simulations have generally supported our predictions. In particular, new regimes (within $0<\sigma<1$ ) have been uncovered in which algebraic solitons demonstrate stable- 
attractor properties when subject to large angular perturbations. Numerical analysis has also provided evidence of algebraic dark-soliton stability.

The new solitons reported here have innate mathematical appeal as exact solutions to generic nonintegrable elliptic equations. Our results are also of physical interest, particularly in photonics, where we propose that Helmholtz soliton theory will play a central role in the design of future integrated-optic devices that exploit non-trivial angular geometries. Indeed, the coexistence of many different solution families (plane waves, hyperbolic and algebraic solitons) could open up the possibility of exciting new multiplexing [21] and interface [22] applications within Helmholtz-nonparaxial configurations.

\section{References}

[1] Dodd R K, Eilbeck J C, Gibbon J D and Morris H C 1982 Solitons and Nonlinear Wave Equations (London: Academic Press)

Remoissenet M 1999 Waves Called Solitons: Concepts and Experiments, $3^{\text {rd }}$ Ed (Berlin: Springer-Verlag)

Infeld E and Rowlands G 2000 Nonlinear Waves, Solitons and Chaos, $2^{\text {nd }}$ Ed (Cambridge: C.U.P.)

[2] Kivshar Y S and Malomed B A 1989 Rev. Mod. Phys. 61763

[3] Klaus M, Pelinovsky D E and Rothos V M 2006 J. Nonlinear Sci. 161

Ablowitz M J and Satsuma J 1977 J. Math. Phys. 192180

[4] Pelinovsky D E and Grimshaw R H J 1997 Phys. Lett. A 229165

Ono H 1976 J. Phys. Soc. Japan 411817

[5] Brunhuber C, Mertens F G and Gaididei Y 2007 Eur. Phys. J B 5757

[6] Fokas A S, Pelinovsky D E and Sulem C 2001 Physica D 152189

Satsuma J and Ablowitz M J 1979 J. Math. Phys. 201496

Watanabe Y and Tajiri M 1998 J. Math. Phys. 67705

Tajiri M, Arai T and Watanabe Y 1998 J. Phys. Soc. Japan 674051

[7] Pelinovsky D E and Sulem C 1998 J. Math. Phys. 396551

Benjamin T B 1966 J. Fluid Mech. 25241

Ono H 1975 J. Phys. Soc. Japan 391082

Meiss J D and Pereira N R 1978 Phys. Fluids 21700

[8] Kennel C F, Buti B, Hada T and Pellat R.1988 Phys. Fluids 311949

[9] Manakov S V, Zakharov V E, Bordag L A, Its A R, and Matveev V B 1977 Phys. Lett. A 63205

[10] Skryabin D V and Yulin A V 2006 Phys. Rev. E 74046616

[11] Belenov E M and Poluéktov 1969 Sov. Phys. JETP 28754

Hanamura E 1974 J. Phys. Soc. Jap. 371598

[12] Mihalache D, Truta N, Panoiu N C, and Baboiu D M 1993 Phys. Rev. A 473190

Mihalache D and Panoiu N C 1993 J. Phys. A: Math. Gen. 26, 2679

[13] Hayata K and Koshiba M 1995 Phys Rev. E 511499

Hayata K and Koshiba M 1995 Opt. Lett. 201131

[14] Hayata K and Koshiba M 1994 J. Opt. Soc. Am. B 112581

Hayata K and Koshiba M 1995 Phys. Rev. E 515155

[15] Alatas H, Iskandar A A, Tjia M O and Valkering T P 2006 Phys. Rev. E 73066606

Grimshaw R and Malomed B 1994 Phys. Rev. Lett. 72949

[16] t'Hooft G 1976 Phys. Rev Lett. 378

Polyakov A M 1974 JETP Lett. 20194

[17] Micallef R W, Afansjev V V, Kivshar Y S and Love J D 1996 Phys. Rev. E 542936

[18] Kivshar Y S 1998 Opt. Quant. Electron. 30571

Kivshar Y S and Luther-Davies B 1998 Phys. Rep. 29881

Stegeman G and Segev M 1999 Science 2861518

[19] Zakharov V E and Shabat A B 1972 Sov. Phys. JETP 3462

Satsuma J and Yajima N 1974 Suppl. Prog. Theor. Phys. 55284

Gordon J P 1983 Opt. Lett. 8596

Cohen O, Uzdin R, Carmon T, Fleischer J W, Segev M and Odouov S 2002 Phys. Rev. Lett. 89 133901 
[20] Aceves A B, Moloney J V and Newell A C 1989 Phys. Rev. A 391809 Aceves A B, Moloney J V and Newell A C 1989 Phys. Rev. A 391828

[21] Chamorro-Posada P and McDonald G S 2006 Phys. Rev. E 74, 036609

[22] Sánchez-Curto J, Chamorro-Posada P and McDonald G S 2007 Opt. Lett. 321126

[23] Feit M D and Fleck J A 1988 J. Opt. Soc. Am. B 5633

[24] Chamorro-Posada P, McDonald G S and New G H C 2002 J. Opt. Soc. Am. B 191216

Laine T A and Friberg A T 2000 J. Opt. Soc. Am. B 17751

Blair S 2000 Chaos 10570

Sheppard A P and Haelterman M 1998 Opt. Lett. 231820

Fibich G 1996 Phys. Rev. Lett. 764356

[25] Lax M, Louisell W H and. McKnight W B 1975 Phys. Rev. A 111365

[26] Chi S and Guo Q 1995 Opt. Lett. 201598

[27] Ciattoni A, Crosignani B, Di Porto P, Scheuer J and Yariv A 2006 Opt. Exp. 145517

Ciattoni A, Crosignani B, Mookherjea S and Yariv A 2005 Opt. Lett. 30516

Crosignani B, Yariv A and Mookherjea S 2004 Opt. Lett. 291524

Ciattoni A, Di Porto P, Crosignani B and Yariv A 2000 J. Opt. Soc. Am. B 17809

[28] Mihalache D, Bertolotti M and Sibilia C 1989 Progress in Optics ed. E. Wolf (Amsterdam: Elsevier) 27229

Snyder A W and Mitchell D J 1993 Opt. Lett. 18101

[29] Pushkarov K I, Pushkarov D I and Tomov I V 1979 Opt. Quantum Electron. 11471

Pushkarov K I and Pushkarov D I 1980 Rep. Math. Phys. 1737

[30] Pelinovsky D E, Afanasjev V V and Kivshar Y 1996 Phys. Rev. E 531940

[31] Akhmediev N, Ankiewicz A and Grimshaw R 1999 Phys. Rev. E 596088

[32] Biswas A 2004 Opt. Commun. 235183

[33] Goldstein H 1982 Classical Mechanics $2^{\text {nd }}$ Ed. (Addison Wesley)

[34] Chamorro-Posada P, McDonald G S and New G H C 2001 Opt. Commun. 1921

[35] Chamorro-Posada P, McDonald G S and New GHC 1998 J. Mod. Opt. 451111

[36] Christian J M, McDonald G S and Chamorro-Posada P 2007 J. Phys. A: Math. Theor. 401545

Christian J M, McDonald G S and Chamorro-Posada P 2007 J. Phys. A: Math. Theor. 408601 Corrigendum

[37] Vakhitov M G and Kolokolov A A 1973, Radiophys. Quantum Electron. 16783

Laedke E W, Spatschek K H and Stenflo L 1983 J. Math. Phys. 242764

[38] Christian J M, McDonald G S and Chamorro-Posada P 2007 Phys. Rev. A 76033833

Christian J M, McDonald G S and Chamorro-Posada P 2007 Phys. Rev. A 76033834

Christian J M, McDonald G S and Chamorro-Posada P 2007 Phys. Rev. A 76 049905(E)

[39] Chamorro-Posada P, McDonald G S and New G H C 2000 J. Mod. Opt. 471877

[40] Zakharov V E and Shabat A B 1973 Sov. Phys. JETP 37823

[41] Chamorro-Posada P and McDonald G S 2003 Opt. Lett. 28825

[42] Pelinovsky D E, Kivshar Y S and Afansjev V V 1996 Phys. Rev. E 542015

Barashenkov I V 1996 Phys. Rev. Lett. 771193

Kivshar Y S and Afansjev V V 1996 Opt. Lett. 211135

Kivshar Y S and Królikowski W 1995 Opt. Lett. 201527 